Аскар Сеитов, Магистрант

Южно-Казахстанский Государственный Университет имени М. Ауезова

г. Шымкент, Казахстан E-mail: r.rrr.2016@ inbox.ru

ORCID ID 0000-0003-4271-2607

\title{
ОТРАБОТКА БЫСТРОМЕНЯЮЩИХСЯ ПРОЦЕССОВ
}

Askar Seitov, Magister

M.Auezov South -Kazakhstan State University Shymkent city, the Republic of Kazakhstan

E-mail: r.rrr.2016@inbox.ru

ORCID ID 0000-0003-4271-2607

\section{PRODUCTION OF RAPIDLY CHANGING PROCESSES}

Annotation In this article analyzed by testing of the rapidly changing process, that is way implementation of two principal other cases direct to presentative of the testing of the rapidly changing process. In this case testing of the rapidly changing process, presentative by determinative process, of the secondly cases - externally process. This two presentative defenestration exactly results of reproducing.

Keywords: production of the rapidly changing process $(P C P)$, an ergodic process, determination process, efficiency, concrete method, testing.

Аннотация. В данной статье рассмотрена отработка быстроменяющихся процессов, то есть существуют два принщипиально иных подхода к способу представления БМП. В первом случае модель БМП представляется детерминированным процессом, во втором случае - случайным проиессом. Эти два представления определяют выходные результаты обработки.

Ключевые слова: быстроменяющихся процессов (БМП), эргодичный процесс, детерминированный процесс, эффективность, конкретный метод, отработка.

При обработке быстроменяющихся процессов (БМП) ставятся разнообразные задачи, решение которых преследует различные цели в зависимости от целей работки. Выбор конкретного метода отработки зависит от множества причин и в первую очередь от способа представления БМП. Существуют два принципиально иных подхода к способу представления БМП. В первом случае модель БМП представляется детерминированным процессом, во втором случае - случайным процессом. Эти два представления определяют выходные результаты обработки [1. С.170].

Так, обработка БМП, представленных как детерминированный процесс, требует, по существу, исследования АЧХ. В то же время обработка их при представлении случайным процессом требует обязательного изучения усредненных характеристик. Если в первом случае для обработки достаточно применения амплитудных анализаторов спектра частот, то во втором случае необходимо иметь в наличии различные устройства для квадратирования, суммирования, умножения, усреднения и т.д.

Анализ БМП, основанный на концепции случайности, имеет разнообразные приближения. Основное приближение, которое приходится применять, - это допущение стационарности и эргодичности процесса [2].

Допущение о стационарности процесса позволяет эффективно применять метод корреляционного анализа, с помощью которого можно исследовать различные

Отработка быстроменяющихся Материалы Международной практической интернет-конференции процессов

«Актуальные Проблемы Науки» 
взаимосвязи. Оперативная обработка БМП является наиболее простой и решает задачи первой группы. Она позволяет получить следующие характеристики на определенном временном интервале: среднее значение параметра ту; средневыпрямленное значение В; максимальное значение M; дисперсию D.

Основное достоинство этих характеристик состоит в простоте их получения. Однако выводы, вытекающие из их анализа, являются, слишком поверхностными. Для перечисленных характеристик справедливы некоторые соотношения, позволяющие определить одну характеристику по известным другим. Эти соотношения имеют вид для синусоидальных сигналов:

$$
M=\sqrt{2 \delta} ; \quad B=\sqrt[2]{\frac{2}{\pi}} \sigma
$$

где $y(t)=\mathrm{ym} \sin \mathrm{wt} ; \quad \mathrm{ym}$ - амплитуда сигнала; w- его частота.

Тогда дисперсия сигнала на интервале времени Т определяется из равенства (для стационарного процесса):

$$
\mathrm{D}=\frac{V \frac{2}{n}}{2} ; \quad \sigma=\frac{Y m}{\sqrt{2}}
$$

Максимальное значение сигнала равно его амплитуде и, следовательно:

$$
M=\sqrt{2 \sigma}
$$

Средневыпрямленное значение сигнала В на интервале Т определяется по формуле:

$$
B=\frac{2 y m}{\pi} \text { или } B=\sqrt[2]{\frac{2}{\pi}} \sigma
$$

Рассмотренные характеристики параметров измеряются с помощью аналового устройства (рисунок 1), входящего в состав аппаратуры для измерения спектров [3].

Напряжение, соответствующее реализации параметра, поступает на вход устройства и далее разделяется по трем направлениям.

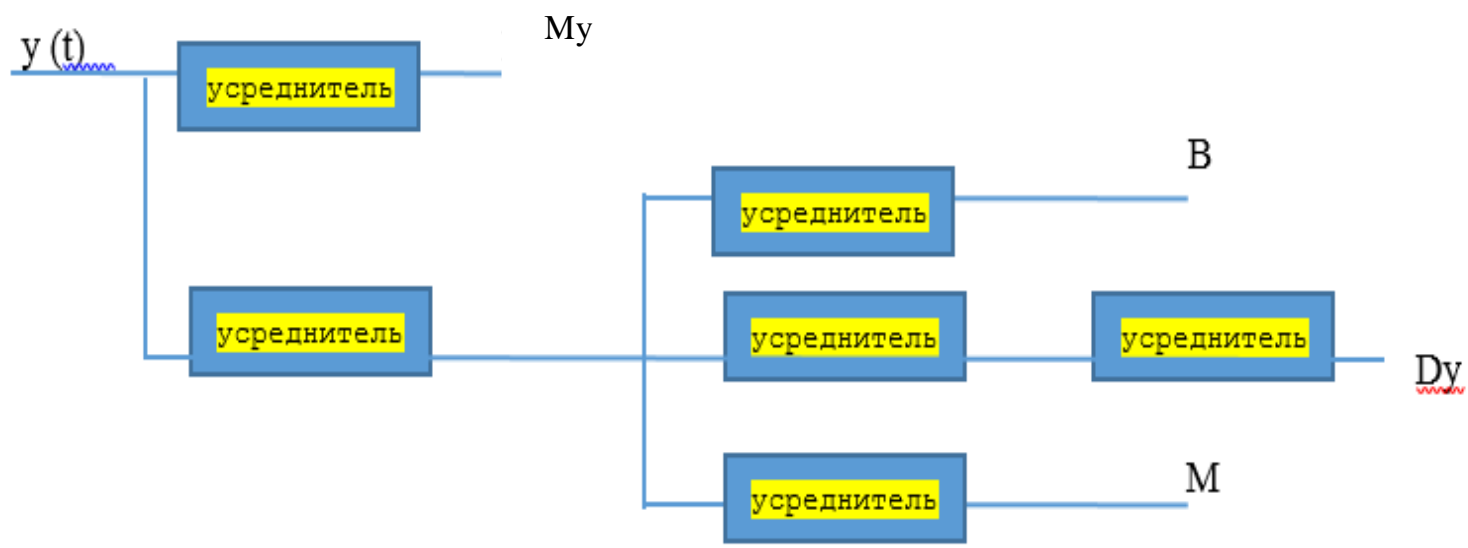

Среднее значение определяется усредняющим устройством, в качестве которого для стационарных эргодических процессов используются интеграторы и фильтры нижних частот. Для нестационарных случайных процессов применяются сглаживающие фильтры, построенные на основе операторов текущего сглаживания. В этих устройствах время 
интегрирования определяется по формуле. Измерение дисперсии стационарного эргодического процесса осуществляется во второй цепочке и сводится к измерению средней мощности центрированного процесса [4].

В этой же цепочке осуществляется измерение средневыпрямленного и максимального значения. Как следует из приведенных выше выражений, для вычисления среднего и дисперсии необходимо знание минимального возможного интервала интегрирования, что предопределяет априорное знание корреляционной функции измеряемого процесса. На практике, как правило, данные о корреляционной функции отсутствуют.

\section{СПИСОК ЛИТЕРАТУРЫ}

1. Есмагамбетов Б.-Б.С., Утепбергенов И.Т. Средства и методы обработки данных. Шымкент: Ну.рлы бейне, 2010. 170 с.

2. 2.О.А.Андреев, Л.Н.Хромов «Учитесь быстро читать»- М.: Просвещение, 1991.

3. 3 .Г.Гранник, Л.А.Концевая, С.М.Бондаренко «Когда книга учит»-М.:Педагогика, 1991.

4. Техника быстрого чтения. Самоучитель по программе Школы Олега Андреева / Олег Андреев. - М.: АСТ: Астрель; Владимир: ВКТ, 2009. 